\title{
Pareto et Ernest Roguin
}

Denis Tappy

\section{OpenEdition \\ Journals}

Édition électronique

URL : http://journals.openedition.org/ress/751

DOI : 10.4000/ress.751

ISSN : 1663-4446

\section{Éditeur}

Librairie Droz

Édition imprimée

Date de publication : 1 juillet 2010

Pagination : 33-44

ISBN : 978-2-600-01449-6

ISSN : 0048-8046

Référence électronique

Denis Tappy, «Pareto et Ernest Roguin », Revue européenne des sciences sociales [En ligne], XLVIII-146 | 2010, mis en ligne le 01 juillet 2013, consulté le 21 avril 2019. URL : http:// journals.openedition.org/ress/751 ; DOI : 10.4000/ress.751 


\section{Denis TAPPY}

\section{PARETO ET ERNEST ROGUIN}

\section{VIE ET GEUVRE D'ERNEST ROGUIN}

Selon une recherche sur Internet, le plus récent ouvrage consacrant des développements substantiels à Ernest Roguin semble être une traduction en portugais, parue il y a quelques mois au Brésil, d'un livre du grand philosophe du droit italien Norberto Bobbio ${ }^{1}$, récemment décédé. Il s'agit essentiellement d'une présentation de l'œuvre de Kelsen, qui s'achève cependant par un chapitre entier consacré au Vaudois, sous le titre révélateur de «Um theorico di direito esquecido: Ernest Roguin» (un théoricien du droit oublié, Ernest Roguin).

Pourtant, à l'instar de Pareto, Roguin a longtemps été considéré comme une figure marquante de l'histoire récente de la Faculté de droit de Lausanne. Il est révélateur à cet égard qu'en 1974 encore, un demi-siècle après sa retraite et plus de 30 ans après sa mort, le volume $\mathrm{V}$ de l'Encyclopédie vaudoise, portant sur les Institutions et destiné à un large public, lui ait consacré un encadré, honneur qu'il ne partageait qu'avec une autre professeur de droit, le grand romaniste Philippe Meylan...2

Depuis, l'étoile de Roguin a sans doute un peu pâli. Son évocation demeure cependant incontournable lorsque l'on évoque Pareto. Il a en effet été, sinon véritablement proche de ce dernier, du moins son plus fidèle ami dans une faculté où Pareto s'est en réalité créé peu de liens. Dans un colloque sur le pan vaudois de sa vie, une présentation sur Pareto et Ernest Roguin s'imposait donc. Giovanni Busino s'est il est vrai déjà livré à une telle évocation dans un article en italien paru il y a près d'un demi-siècle ${ }^{3}$. Cependant, grâce notamment à l'impressionnante édition des œuvres complètes de Pareto et de sa correspondance publiée

1 Cf. Direito e poder (Nilson Moulin trad.), Sao Paulo, 2008. L'ouvrage de Norberto Bobbio ainsi traduit est paru initialement en italien sous le titre Diritto e Potere. Saggi su Kelsen, Naples, 1992. Il comprend un chapitre 10 entièrement consacré à Roguin (Un dimenticato theorico del diritto: Ernest Roguin, pp. 193 ss), qui reprend un article publié par le même Bobbio une quinzaine d'années auparavant (Un dimenticato theorico del diritto: Ernest Roguin, in Scritti in onore di Salvatore Pugliatti, vol. 4: Scritti storico-filosofici, Milan, 1978, pp. 43 ss).

2 Cf. Encyclopédie illustrée du Pays de Vaud, volume V: Les institutions ou le Pouvoir chez les Vaudois (Pierre Cordey dir.), Lausanne, 1974, p. 184.

3 Cf. Giovanni Busino, Ernest Roguin e Vilfredo Pareto, «Cahiers Vilfredo Pareto», 1964, pp. 189 ss, et in Euvres complètes (citées ci-après $O C$, suivi du numéro du tome en chiffres romains) de Vilfredo Pareto, (Giovanni Busino dir.), 32 vol., Droz, Genève 1964-2005, tome XIX/2, 1975, pp. 1232 ss. 
sous sa direction jusqu'en $2005^{4}$, il est devenu possible depuis de préciser encore, sur l'un ou l'autre point, le tableau des relations entre les deux hommes. Il convient cependant de commencer en évoquant de façon générale la carrière et l'œuvre de Roguin 5 .

Ernest Roguin (1851-1939) est un pur Vaudois, né à Yverdon dans un milieu aisé et déjà tourné vers le droit. Son père Jules Roguin (1823-1908), avocat de formation, fera une brillante carrière successivement politique, judiciaire, puis académique, puisqu'il sera tour à tour syndic d'Yverdon, député au Grand Conseil vaudois, conseiller d'Etat, conseiller aux Etats et juge fédéral (il fait partie des premiers magistrats élus dans ce tribunal lorsqu'il devient permanent en 1874), pour finir par devenir professeur de droit public à l'Université de Genève ${ }^{6}$.

Ernest Roguin, de son côté, a fait des études de droit à ce qui n'était encore que l'Académie de Lausanne. Il y soutient en 1874, soit l'année même où Jules Roguin devient juge fédéral, une thèse de doctorat sur le droit international privé en matière de successions ${ }^{7}$. Conformément à l'usage de l'époque, il complète sa formation par un séjour dans une université allemande, à Leipzig. Il embrasse ensuite la carrière diplomatique, qui le conduit jusqu'à un poste de conseiller à la légation suisse de Paris.

Il quitte ce poste en 1884 lorsqu'il est nommé professeur extraordinaire de droit comparé à la Faculté de droit de Lausanne, comme successeur d'Ernest Lehr. Il y enseignera jusqu'en 1926. C'est l'époque où la faculté en question cherche à diversifier ses enseignements en demandant à ses enseignants, faute de moyens pour en engager davantage, de multiplier leurs cours dans des domaines parfois très variés ${ }^{8}$. Roguin donnera ainsi au fil des années des cours de droit comparé, mais aussi de droit civil, de droit international privé et d'introduction au droit ...

En sus desdits enseignements, il publie, beaucoup pour une époque où certains rencontraient de réelles difficultés pour se faire éditer. A côté d'articles ou d'ouvrages de circonstances, les œuvres de Roguin s'inscrivent dans trois directions principales:

4 Des lettres de Pareto ayant échappé aux éditeurs des œuvres complètes (dont trois destinées à Roguin et d'autres qui parlent notamment de lui) ont encore été publiées par Pier Carlo Della Ferrera, Appendice documentaria. 63 lettere dal Fondo Vilfredo Pareto della Banca Popolare di Sondrio, in Vilfredo Pareto (1848-1923). L'uomo e lo scienziato (Gavino Manca dir.), Milan, 2002, pp. 27 ss.

$5 \mathrm{Si}$ aucune autre référence n'est donnée, les renseignements généraux d'ordre biographique qui suivent sont tirés de Olivier Robert et Francesco Panese, Dictionnaire des professeurs de l'Université de Lausanne, Université de Lausanne, 2000, sp. pp. 1092 ss.

6 Jules Roguin fut même un candidat sérieux à une élection au Conseil fédéral, obtenant 52 voix lors de l'élection en 1875 qui vit finalement le Neuchâtelois Numa Droz succéder à Paul Cérésole, cf. Urs Altermatt, Conseil fédéral. Dictionnaire biographique des cents premiers Conseillers fédéraux, Yens, Cabédita, 1993, pp. 219 ss. Il rejoignit la Faculté de droit de Genève lorsqu'il quitta le Tribunal fédéral, en 1890. Le père fut donc nommé professeur après le fils et ils enseignèrent simultanément, l'un à Genève, l'autre à Lausanne! Selon une lettre de Pareto à Pantaleoni du 23 mars 1897, ils n'avaient d'ailleurs pas les mêmes opinions juridiques, cf. $O C$ XXVIII/2, nº 272, pp. 56 ss. Lorsque nous parlerons ci-après de Roguin sans donner de prénom, c'est toujours à Ernest Roguin que nous nous référerons.

7 Essai sur la succession en droit international privé: spécialement au point de vue des relations entre les divers cantons suisses et entre la France et la Suisse, Paris, 1874, 134 p.

8 Cf. Laurent Tissot, Politique, société et enseignement supérieur dans le canton de Vaud. L'université de Lausanne. 1890-1916, Lausanne, Payot, 1996, pp. 224 ss. 
- la philosophie du droit et la théorie juridique, à laquelle il consacre en 1889 un premier ouvrage qui rétrospectivement apparaît peut-être comme son œuvre maîtresse, La Règle de droit ${ }^{9}$. Il reprendra et développera les mêmes conceptions en 1923 dans un traité totalisant plus de 1000 pages au total, la Science juridique pure $^{10}$. Il y soutient une conception positiviste d'un droit se voulant pleinement scientifique, d'une manière qui n'est pas sans parenté avec la Reine Rechtslehre de Kelsen, quoique aucun des deux n'ait apparemment connu l'œuvre de l'autre ${ }^{11}$;

- le droit comparé, qui est toujours resté un de ses domaines d'enseignement, avec en particulier un traité en 7 volumes de 1904 à $1912^{12}$;

- à la fin de sa vie, un projet de traité de sociologie, publié alors que Roguin avoisinait les 80 ans et qui en est resté en réalité à des prolégomènes, alors même que la partie qui a vu le jour, entre 1928 et 1932, compte 5 volumes et près de 4'000 pages au total ${ }^{13}$ !

En sus de ses enseignements et de son œuvre doctrinale, Ernest Roguin a eu d'importantes fonctions internationales. Entre 1893 et 1904, il a en particulier représenté à de multiples reprises la Confédération, comme comparatiste et spécialiste du droit international privé, aux conférences de la Haye qui discutaient alors des Conventions portant le nom de cette ville. A partir de 1912, il fut aussi délégué aux conférences de droit international de Londres, cette fois pour le compte de l'Empire ottoman. Cela explique sans doute qu'il ait joui d'une très grande notoriété et qu'il ait été couvert d'honneurs, dont trois doctorats honoris causa (Genève, Zurich et Lyon), la légion d'honneur, une décoration ottomane (l'ordre du Médjidié) et une autre néerlandaise (l'ordre l'Orange-Nassau).

L'œuvre d'Ernest Roguin a naturellement subi l'épreuve du temps. Son Traité de droit comparé ne peut plus être utile aujourd'hui qu'à un historien du droit. Sa Sociologie a souvent été perçue comme un fourvoiement de fin de vie: Norberto Bobbio n'écrivait-il pas en 1981 que cette œuvre avait «sombré dans un silence quasi sépulcral », dont elle ne lui paraissait pas mériter de sortir, concluant par ces mots:

Le passage de l'auteur de La Règle du droit à la sociologie fut une véritable culbute, de sorte que s'il est juste de lui attribuer en tant que théoricien du droit une place d'honneur (...) le voile de l'oubli qui l'a recouvert en sa qualité de sociologue ne sera, je crois, jamais plus soulevé ${ }^{14}$.

${ }^{9}$ La règle de droit: analyse générale, spécialités, souveraineté des Etats, assiette de l'impôt, théorie des statuts, système des rapports de droit privé, précédé d'une introduction sur la classification des disciplines par Ernest Roguin, Lausanne et Paris, 1889.

${ }^{10}$ La science juridique pure par Ernest Roguin, 3 vol., Paris et Lausanne, 1923.

${ }^{11} \mathrm{Cf}$. François Guisan, La science juridique pure, Roguin et Kelsen, «Revue de droit suisse», 1940, II, pp. 207 ss.

${ }^{12}$ Traité de droit civil comparé par Ernest Roguin, 7 vol., Paris et Lausanne, 1904-1912.

13 Sociologie par Ernest Roguin, 5 vol., Lausanne, 1928-1932. L'intérêt de Roguin pour la sociologie était toutefois ancien et dans le premier volume de son traité de droit civil comparé il plaidait déjà pour une approche du droit impliquant des aspects sociologiques, cf. Busino, Roguin e Pareto, art. cit., O.C., XIX/2, p. 1239.

${ }^{14}$ Cf. Norberto Bobbio, Le Vaudois Ernest Roguin, sociologue et théoricien du droit (trad. Alain Dufour), «Revue européenne des sciences sociales», 1981, pp. 121 ss (spécialement p. 140). 
Un tel jugement est peut-être excessivement dépréciatif. Il semble effectivement que les 5 volumes en question n'aient guère apporté au développement scientifique de la sociologie. Si on a la patience de les ouvrir, on y trouve cependant, surtout dans les deux derniers, un peu en vrac de nombreux développements sur ce que pensait Roguin ou ce qui l'intéressait, y compris des problèmes de physique, de politique, d'histoire, etc. Il y a par exemple de nombreuses pages sur des points d'historiographie, de la chute de l'Empire romain à l'affaire Dreyfus en passant par la naissance de la Confédération, sur des questions d'épistémologie scientifique, à propos par exemple de la théorie de la relativité d'Einstein, sur des philosophes récents ou contemporains, comme Nietzsche ou Bergson, ou encore sur des problèmes politiques de l'entre-deux-guerres. Leur étude ne serait pas dépourvue d'intérêt, au moins dans le cadre d'une étude centrée sur l'auteur lui-même, sa personnalité, ses lectures, etc., voire dans une perspective prosopographique, comme témoignage sur un représentant des milieux universitaires vaudois du début du $\mathrm{XX}^{\mathrm{e}}$ siècle.

C'est cependant effectivement par le premier volet de son œuvre que Roguin a gardé dans la pensée juridique une place qui dépasse les frontières de notre pays et celle du monde francophone (même si c'est évidemment en Suisse romande que sa notoriété est restée la plus grande, plusieurs de ses collègues lausannois ${ }^{15}$ ou romands ${ }^{16}$ l'ayant régulièrement cité, voire lui ayant consacré des travaux ${ }^{17}$ jusqu'au deuxième tiers du $\mathrm{XX}^{\mathrm{e}}$ siècle): sans exhaustivité, on peut relever l'intérêt pour son œuvre manifesté en Italie, où une thèse de philosophie du droit lui a été consacrée à Turin en $1957^{18}$. Sa Règle de droit, qui avait été traduite en espagnol il y a plus de 100 ans $^{19}$, vient par ailleurs d'être rééditée dans cette langue ${ }^{20}$. Dans les pays germanophones, sa pensée avait été signalée entre deux guerres déjà par un article de l'Autrichien Schreier ${ }^{21}$. En France enfin, Ernest Roguin reste par exemple évoqué dans deux articles, relatifs respectivement à la codification et au positivisme, du récent Dictionnaire de la culture juridique ${ }^{22}$.

${ }^{15}$ Cf. par exemple Karl-Heinz Neumayer, Les droits sans sujet, «Revue internationale de droit comparé », 12 (1960), pp. 342 ss., et Otto Riese, Une juridiction supranationale pour l'interprétation du droit unifié?, «Revue internationale de droit comparé», 13 (1961), pp. 717 ss.

${ }^{16}$ La pensée de Roguin est particulièrement évoquée par Claude Du Pasquier dans son Introduction à la théorie générale et à la philosophie du droit, parue initialement à Paris et Neuchâtel en 1937 et régulièrement rééditée jusqu'à nos jours.

17 Voir en particulier les études de Guisan, citée à la note 11 ci-dessus, et de Busino, citée à la note 3 ci-dessus, ainsi que du même Giovanni Busino, Ernest Roguin tra diritto e sociologia, in Sociologia e storia. Elementi per un dibattito, Naples, Guida, 1975, pp. 427 ss.

${ }^{18}$ Roberto Codazza, Il problema della scienza giuridica pura in E. Roguin, Universita du Torino, Facolta di Giurisprudenza, tesi in filosopfia del Diritto, 150 p. dact., 1957. Nous sommes particulièrement reconnaissant à M. Codazza de nous avoir fait parvenir une copie de son travail, qui peut désormais être consulté à la Bibliothèque de la Fondation Edouard Fleuret, à Dorigny. La connaissance de l'œuvre de Roguin en Italie doit évidemment beaucoup aux travaux de Norberto Bobbio cités aux notes 1 et 14 ci-dessus.

${ }^{19}$ Cf. Ernest Roguin, Las Reglas juridicas (J.-M. Navarro de Palencia trad.), Madrid, 1904.

${ }^{20}$ Rééd. Analecta editorial, Pampelune, 2005.

${ }^{21}$ Fritz Schreier, Eine französische reine Rechtslehre, «Revue internationale de la théorie du droit», II (1927-1928), pp. 57 ss.

${ }^{22}$ Cf. Dictionnaire de la culture juridique (Denis Alland et Stéphane Rials dir.), Paris, Presses universitaires de France, 2003. 
Sur le plan personnel, ses contemporains ont généralement décrit Ernest Roguin, dont, sur commande de l'Université, le peintre Bieler a réalisé en 1928 un portrait saisissant ${ }^{23}$, comme un homme bon vivant et aimable. Malgré des frictions occasionnelles qui seront évoquées plus loin, il a en tout cas réussi à ne pas se brouiller avec ledit Pareto, contrairement à d'autres. Au contraire de son père, il n'a jamais fait de carrière politique, même s'il avait des opinions solidement à droite. Il s'opposait en particulier avec force aux tendances socialistes, ce qui contribua sans doute à le rapprocher de Pareto $^{24}$. Il eut à la fin de sa vie de fortes sympathies pour le mouvement maurassien et a contribué à la vocation maurassienne de certains étudiants, en particulier les fondateurs de la Ligue vaudoise Marcel Regamey, Alphonse Morel et Victor de Gautard ${ }^{25}$. Dans sa Sociologie, il cite effectivement souvent Charles Maurras, Léon Daudet et le journal L'Action française, en général de manière élogieuse, même s'il lui arrive aussi de les critiquer.

Durant sa très longue carrière à la Faculté de droit de Lausanne, Roguin s'est beaucoup investi pour le développement de celle-ci. On ne doit pas accorder à cet égard trop d'importance au fait qu'il en a été doyen pendant deux périodes distinctes $^{26}$, dont la première comprenait l'année 1890 qui vit la transformation de l'Académie en université: à l'époque il y avait en effet si peu de professeurs que c'était une charge relativement légère, attribuée par un pur tournus. En revanche, il faut souligner la constance avec laquelle Roguin s'est engagé en faveur de l'extension et du renouveau de ladite faculté: il a été pour beaucoup, nous y reviendrons, dans les démarches qui ont finit par aboutir en 1902 à la création d'une filière d'études en Sciences sociales, dont est issue l'Ecole des SSP ${ }^{27}$. Il a d'ailleurs présidé cette nouvelle école, qui restait alors rattachée à la Faculté de droit, jusqu'en 1917. Par la suite, il a aussi contribué à la création, avec un rattachement analogue, de l'Ecole des $\mathrm{HEC}^{28}$.

Dans un domaine plus strictement juridique, Roguin a lutté pour que la Faculté de droit de l'Université de Lausanne cesse d'être essentiellement destinée à un public local et pour que dans les enseignements dispensés, qu'il aurait voulu diversifier, la science du droit l'emporte sur l'exégèse du droit positif ${ }^{29}$. A ce titre, il a notamment poussé à la fin du XIX ${ }^{\mathrm{e}}$ siècle à l'institution et à la stabilisation de cours de droit allemand et de droit romain enseignés en allemand pour attirer à

${ }^{23}$ Pour une reproduction en noir et blanc et un descriptif de ce tableau, cf. François Belperrin et Patrick Schaefer, Les portraits professoraux de la Salle du Sénat, Palais de Rumine, Université de Lausanne, 1987, pp. $102 \mathrm{~s}$.

${ }^{24}$ Cf. Busino, Roguin e Pareto, art. cit., O.C., XIX/2, p. 1239.

${ }^{25}$ Cf. notamment François Wisard, L'université vaudoise d'une guerre à l'autre. Politique, finances, refuge, Lausanne, Payot, 1998, pp. 183 ss. Roland Butikofer, Le refus de la modernité. La Ligue vaudoise: une extrême droite et la Suisse (1919-1945), Lausanne, Payot, 1996, pp. 75 ss.

${ }^{26}$ Soit de 1890 à 1892 , puis de 1902 à 1904.

${ }^{27}$ Cf. à ce sujet notamment Tissot, Politique, société et enseignement supérieur, op. cit., pp. 240 ss et Fiorenzo Mornati, La création de l'Ecole des Sciences sociales à Lausanne (1890-1902): le rôle de Pareto, «Les constitutions vaudoises. 1803-2003. Miroir des idées politiques» (Olivier Meuwly dir.), Bibliothèque historique vaudoise 123, Lausanne, 2003, pp. 353 ss.

${ }^{28} \mathrm{Cf}$. à ce sujet notamment Tissot, Politique, société et enseignement supérieur, op. cit., pp. $250 \mathrm{ss}$.

29 Ibidem, p. 223 ss. 
Lausanne de jeunes juristes allemands ${ }^{30}$. Dans cet esprit d'ouverture il a aussi lutté, lors de la création d'un cours de droit administratif en 1902, pour qu'on fasse appel à un étranger (ce sera le Français Antoine Rougier) plutôt que de privilégier une candidature locale ${ }^{31}$. En revanche, s'il était encore loin de sa retraite lors de la création en 1909, toujours au sein de la Faculté de droit, de l'Institut de police scientifique, nous n'avons pas réussi à savoir s'il s'était intéressé à ce nouveau développement...

\section{RELATIONS DE ROGUIN AVEC PARETO}

Les deux hommes ne se sont probablement connus qu'au printemps 1893 , lorsque Pareto est arrivé à Lausanne pour reprendre l'enseignement de Walras. On sait qu'il correspondait avec ce dernier depuis 1891 et qu'il était déjà venu sur les bords du Léman en septembre de ladite année pour le rencontrer ${ }^{32}$, mais il ne semble pas que lors de ce passage il ait fait la connaissance d'autres membres de la Faculté de droit ${ }^{33}$. Roguin, qui venait de transmettre le décanat à Louis Grenier, ne semble pas non plus avoir joué de rôle particulier dans la nomination de Pareto, intervenue en avril $1893^{34}$. Ils ont dû faire connaissance en mai 1893 , au moins lors de la leçon inaugurale donnée par ce dernier le 12 du même mois déjà ${ }^{35}$.

Ils semblent s'être rapidement liés. En automne 1893 déjà, Roguin introduit par exemple Pareto dans le Cercle littéraire de Lausanne ${ }^{36}$. Par la suite, ils ont collaboré notamment en 1895 lors de démarches communes de la Faculté de droit et de celle des lettres tendant déjà à introduire un diplôme en sciences sociales ${ }^{37}$. Ces démarches, même restées alors infructueuses, ont dû renforcer leurs liens.

L'amitié qui se développe ainsi va durer jusqu'à la mort de Pareto en 1923. Elle contribue à créer pour ce dernier des liens dans une faculté au sein de laquelle il restera pour le surplus assez peu intégré, n'entretenant avec la plupart de ses

${ }^{30}$ Ibidem, p. 228 ss. Voir aussi les revendications, soutenues par Roguin, alors doyen de la Faculté de droit, du professeur de droit romain également chargé depuis 1886-1887 d'un cours particulier de droit allemand Henri Erman à l'adresse du conseiller d'Etat Ruffy, à l'occasion de la transformation de l'Académie en Université en 1890, cf. Marie France Zeller et Pierre-Alain Liard, Les Professeurs de l'Université de Lausanne, 1890-1939, Université de Lausanne, 2005, pp. 36 s. et 290 ss (publication des lettres d'Erman et de Roguin).

31 Tissot, Politique, société et enseignement supérieur, op. cit., p. 226.

${ }^{32} \mathrm{Cf}$. Giovanni Busino, Vilfredo Pareto à travers sa correspondance, in L'Ecole de Lausanne de Léon Walras à Pasquale Boninsegni (Giovanni Busino et Pascal Bridel éd.), Université de Lausanne, 1987, p. 39, et les lettres relatives à la venue de Pareto à Lausanne en 1891, O.C., XIX/1, $\mathrm{n}^{\text {os }} 16$ ss, pp. 160 ss.

${ }^{33}$ Durant les démarches qui suivent sa candidature à la succession de Walras, il dit ne connaître à Lausanne personne d'autre que ce dernier, cf. Busino, «Pareto à travers sa correspondance», op. cit., p. 40 .

${ }^{34}$ Busino, Pareto à travers sa correspondance, art. cit., pp. $39 \mathrm{~s}$.

35 Ibidem, p. 42.

${ }^{36}$ Cf. O.C., XX, Jubilé du professeur V. Pareto, Genève, Droz, 1975, préface de Jean-Charles Biaudet, p. 9.

${ }^{37}$ Cf. Tissot, Politique, société et enseignement supérieur, op. cit., p. 241 ss; Mornati, Le rôle de Pareto, art. cit., pp. 358 ss. 
collègues juristes que des relations très formelles. Parmi les autres professeurs de droit lausannois, on ne peut guère citer que le romaniste allemand Henri Erman avec qui Pareto ait sympathisé avant qu'il ne parte pour Münster en $1902^{38}$, ainsi que par la suite le pénaliste et internationaliste André Mercier, nommé en $1901^{39}$. Roguin et Mercier seront d'ailleurs les seuls membres de la Faculté de droit présents aux obsèques de Pareto en août $1923^{40}$.

Il est difficile de savoir jusqu' où allait cette amitié. Une désignation comme «il mio amico Roguin» dans une lettre de Pareto ${ }^{41}$, ne prouve évidemment pas grand-chose et il ne s'est probablement jamais agi d'une véritable intimité. Elle dépassait cependant la simple bonne entente entre collègues. On trouve par exemple dans la correspondance qu'ils ont régulièrement échangée durant les années de Céligny (tant que Pareto a habité Lausanne même, Roguin et lui avaient moins de raisons de s'écrire) des éléments personnels ou privés, au sujet de chats, de champignons ou encore d'excursions en montagne ... Même à la fin de la vie de Pareto, alors que ce dernier se déplaçait de moins en moins volontiers jusqu'à Lausanne, ils ont continué à se voir, Roguin venant assez régulièrement à Céligny, où Pareto l'a même souvent invité à dormir.

On sait aussi certaines choses par des correspondances de tiers. Maffeo Pantaleoni, le plus ancien et le plus proche ami de Pareto, n'a pas toujours apprécié Roguin. Dans une lettre de 1908 à Mme Régis, la nouvelle compagne de Pareto, il écrit en tout cas:

Ne dégoûtez pas Roguin. Vous ne l'aimez pas et vous avez raison. Mais n'en faites pas un ennemi. Après tout, il amuse quelque fois Vilfredo et s'il se voyait mis de côté, étant donné son caractère de vipère, il pourrait nuire ${ }^{42}$.

Il est vrai que, dans d'autres correspondances, Pantaleoni est plus laudatif sur Roguin: en particulier, après les cérémonies du jubilé de 1917, il rendra hommage à la justesse et l'habileté avec lesquelles ce dernier avait su rendre compte des travaux sociologiques de Pareto $^{43}$.

${ }^{38}$ Cf. O.C., XX, p. 10. Pareto discutait notamment avec Erman de questions touchant l'histoire antique, qu'il appréciait et invoquait fréquemment dans ses cours, et c'est grâce à l'intervention du romaniste allemand, alors doyen de la Faculté de droit, que fut trouvée en 1900 une solution permettant à Pareto de rester professeur avec une charge d'enseignement allégée après sa première « démission », cf. Biaudet, Préface, art. cit., in O.C., XX, p. 12 et Busino, Pareto à travers sa correspondance, art. cit., pp. 48 s. Dans son discours de remerciement lors du jubilé de 1917, Pareto évoquera publiquement sa communauté d'idées avec Erman, in O.C., XX, p. 67.

${ }^{39}$ Cf. Biaudet, Préface, art. cit., in O.C., XX, p. 10. Outre des rapports amicaux, Pareto eut aussi des échanges avec Mercier au sujet de la législation pénale, notamment lorsqu'en 1921-1922 il s'intéressa au projet Ferri de réforme du Code pénal italien, cf. Giovanni Busino, Vilfredo Pareto, la scuola positiva ed il progetto Ferri di codice penale, in O.C., XIX/2, pp. 1192 ss. Dans une lettre du 25 décembre 1916 à Pantaleoni, il décrit ledit Mercier comme une excellent ami, fidèle et loyal, in O.C., XXVIII/3, n 646, pp. 201 ss.

${ }^{40} \mathrm{Cf}$. Busino, Pareto à travers sa correspondance, art. cit., p. 31. Nous ignorons les raisons de l'absence du doyen de 1923, soit le Français Rougier mentionné plus haut, qui n'était peut-être simplement pas en Suisse en cette période estivale.

${ }^{41}$ Cf. par exemple lettre du 2 février 1902 à A. Linaker, O.C., XIX/1, n 298, p. 442 s.

${ }^{42}$ Cf. lettre de Pantaleoni à Jeanne Régis, 15 août 1908, O.C., XVIII/3, nº 14, p. 375.

${ }^{43}$ Cf. Bobbio, Le Vaudois Ernest Roguin, art. cit., p. 124 n. 10. 
Il est bien connu que Pareto n'était pas un caractère facile et qu'il a fini brouillé avec beaucoup de gens qu'il avait d'abord apprécié, comme Millioud, Racca ou Boninsegni ${ }^{44}$, voire Walras ${ }^{45}$. Il faillit en aller de même avec Roguin. Une première crise est liée à l'affaire de la soutenance de thèse de Maria Kolabinska en 1912, dont il sera question ultérieurement. Dans des lettres immédiatement postérieures, Pareto ne l'appelle plus son cher ami, mais «Mr R.» ${ }^{46}$, voire «le pauvre Monsieur Roguin »... ${ }^{47}$ Les choses s'arrangèrent rapidement, mais le 16 juin 1916 c'est Roguin qui laisse transparaître une certaine aigreur, en relevant lors d'une séance relative à l'Ecole des sciences sociales que Pareto paraît se désintéresser de cette dernière et n'a pas même jugé bon de le tenir informé, comme président de cette Ecole, des cours qu'il donnait en sociologie ${ }^{48}$. Il y aura aussi des tensions sur lesquelles nous reviendrons au moment de l'organisation du jubilé de 1917. Elles ne tarderont pas cependant à s'apaiser.

Pareto a parfois tiré un profit institutionnel de cette amitié. Ainsi, lors des événements qui conduisirent en 1903 à l'éviction de Racca de la Faculté de droit, Roguin, qui était à nouveau doyen de cette dernière, soutiendra pleinement la position de Pareto ${ }^{49}$. Les bons rapports entre les deux hommes ont aussi profité à l'institution, par exemple lorsqu'en 1907 Pareto fera don à l'Université de Lausanne de sa bibliothèque, cela par l'intermédiaire de Roguin ${ }^{50}$. Ils ont évidemment été particulièrement bénéfiques pour le développement des enseignements en sciences sociales. On a déjà mentionné leur participation conjointe aux travaux de 1895 à ce sujet. Ils seront aussi associés dans les démarches en 1901-1902 qui finiront par aboutir à la création d'une Ecole des sciences sociales dans le cadre de

${ }^{44}$ Cf. Biaudet, Préface, art. cit., in O.C., XX, p. 13 ss. Sur la carrière et l'œuvre de Millioud, cf. notamment Daniel Christoff, L'enseignement de la philosophie à l'Académie et dans les premiers temps de l'Université, in La Philosophie dans la Haute Ecole de Lausanne, 1542-1955, Université de Lausanne, 1987, pp. 76 ss; sur celles de Racca et de Boninsegni, cf. Giovanni Busino, Le successeur de Walras et de Pareto: Pasquale Boninsegni et Vittorio Racca (1876-1957), in L'Ecole de Lausanne, op. cit., pp. 83 ss et 95 ss.

${ }^{45}$ Sur l'éloignement survenu entre Walras et Pareto, dont la dernière lettre à son prédécesseur est de 1901, cf. Georges-Henri Bousquet, Pareto (1848-1923). Le savant et l'homme, Lausanne, Payot, 1960, pp. 50 ss. Invoquant des problèmes de santé, Pareto ne participera pas à la célébration du jubilé de Walras en 1909. Lorsque quelques années plus tôt ce dernier voulut postuler le prix Nobel de la paix, c'est à Roguin et non à Pareto qu'il demanda de présenter sa candidature, cf. en dernier lieu Agnar Sandmo, Retrospectives: Léon Walras and the Nobel Peace Prize, «Journal of Economic Perspectives », 21 (2007), pp. 217 ss. De même, quand Boninsegni souhaita être présenté à Walras en 1908, il demanda ce service à Roguin et non à Pareto, bien qu'il fût le suppléant de ce dernier, cf. Giovanni Busino, Le successeur de Walras et de Pareto: Pasquale Boninsegni, in L'Ecole de Lausanne, op. cit., p. 88.

${ }^{46}$ Cf. Lettre à Boven du 25 mai 1912 et annexe, O.C., XIX/2, n 737 p. 768 ss.

${ }^{47}$ Cf. Lettre à Boven du 14 mai 1912, O.C., XIX/2, nº 736, p. 768. Des années plus tard, lors des tensions qui précédèrent le jubilé de 1917, Pareto traite Roguin de «gros serpent à sonnette» (cf. lettre à Pantaleoni du 9 janvier 1917, O.C., XXVIII/3, nº 647, pp. 203 ss: quel grosso serpente a sognali che ha nome Roguin).

${ }^{48} \mathrm{Cf}$. Tissot, Politique, société et enseignement supérieur, op. cit., pp. $262 \mathrm{~s}$.

49 Sur cette affaire, cf. surtout Giovanni Busino, Vittorio Racca, art. cit., pp. 107 s. et les lettres publiées in $O . C$., XIX/1, $\mathrm{n}^{\text {os }} 371 \mathrm{ss}$, pp. $500 \mathrm{ss}$.

${ }^{50} \mathrm{Cf}$. Busino, Pareto à travers sa correspondance, art. cit., pp. $53 \mathrm{~s}$. 
ladite faculté ${ }^{51}$. Une fois la nouvelle filière mise en place, ils seront les deux premiers représentants de la Faculté de droit dans la commission chargée d'admettre les étudiants, administrer les examens et délivrer les diplômes ${ }^{52}$.

Roguin prendra ce rôle très à cœur, et présidera on l'a dit la jeune école jusqu'en 1917. Pareto au contraire souhaitait, depuis les dernières années du XIX siècle déjà, se désinvestir d'une grande partie de ses responsabilités relatives à l'enseignement, pour se consacrer à l'écriture. Il a peut-être eu tendance à se désintéresser de l'Ecole des sciences sociales nouvellement créée, comme on a vu Roguin le déplorer en 1916. Il intervint néanmoins lors des discussions qui aboutirent à la création en 1911, également dans le cadre de la Faculté de droit, de l'Ecole des Hautes études commerciales, trouvant que les développements proposés dans ce cadre recevaient une tournure trop pratique: il aurait voulu conserver une orientation essentiellement théorique et semble avoir reproché cette tendance utilitaire à Millioud surtout, mais aussi à Roguin ${ }^{53}$. Il n'empêche que l'alliance de ce dernier et de Pareto, et le soutien au moins passif de celui-ci, grâce notamment à son immense notoriété, ont joué un rôle important dans la naissance des futures facultés des SSP et des HEC ...

Les échanges entre Roguin et Pareto sur le plan scientifique n'ont en revanche pas toujours été sans nuage. Tout avait pourtant bien commencé: Pareto s'était intéressé aux publications de Roguin, auxquelles il se réfère parfois dans ses propres ouvrages ${ }^{54}$. Il recourait parfois aussi aux compétences juridiques de son ami pour ses affaires privées. Ainsi, dans le serpent de mer que représentait la question de la dissolution de son premier mariage, il avait envisagé de faire prononcer un divorce selon le droit russe, vu les origines de son épouse, née Bakounine. En 1902, il déclare à son avocat italien avoir consulté à cet égard Roguin, dont il exalte les qualités de juriste ${ }^{55}$.

A l'inverse, le juriste lausannois suivait les travaux de Pareto. Celui-ci écrira par exemple à Pantaleoni que Roguin déclarait son Cours d'économie politique

${ }^{51}$ Cf. Tissot, Politique, société et enseignement supérieur, op. cit., pp. 244 ss; Mornati, Le rôle de Pareto, art. cit., pp. 361 ss. Le rôle effectif qu'a joué Pareto dans la création de la filière débouchant sur la délivrance d'un diplôme en sciences sociales est controversé. Mornati l'estime plus modeste qu'on ne l'a souvent pensé et considère que c'est davantage la présence à Lausanne et le renom du savant italien qui a favorisé cette création qu'un engagement de l'intéressé dans les démarches ellesmêmes qui y ont conduit.

${ }^{52}$ La Faculté des lettres était de son côté représentée initialement par Millioud et Rossier (plus tard remplacé par Sirven), alors qu'après quelques années, Pareto laissa sa place dans cette commission à Boninsegni, cf. Tissot, Politique, société et enseignement supérieur, op. cit., p. 249 n. 237 et p. 255 n. 257; Biaudet, Préface, art. cit., in O.C., X, p. 12 et 14.

${ }^{53}$ Cf. Tissot, Politique, société et enseignement supérieur, op. cit., pp. 252 ss, sp. 257.

${ }^{54}$ La Règle de droit est notamment citée comme «une des œuvres les plus remarquables de notre époque sur cette matière» dans le Cours d'économie politique, paru en 1896-1897, \$ 34 n. 2, cf. O.C., I, p. 15. En 1898, Pareto avait prêté son exemplaire à Pantaleoni et dû le lui réclamer plusieurs fois, cf. O.C., XXVIII/2, n ${ }^{\text {os }} 345,347$ et 354, pp. 177 ss. En 1912, malgré la crise de l'affaire Kolabinska, Pareto remerciait Roguin de l'envoi de ses livres et l'informait qu'il citait ceux-ci comme des modèles dans son Traité de sociologie générale, cf. lettre du 14 juillet 1912, éd. par Della Ferrara, Appendice, art. cit., $\mathrm{n}^{\circ} 35$, pp. 347 ss. Effectivement, La Règle de droit et le Traité de droit civil comparé sont mentionnés aux $\S 839$ et 840 dudit traité (O.C., XII, pp. $448 \mathrm{~s}$ ).

${ }^{55}$ Lettre à Vittorio Calosi du 28 janvier 1902, O.C., XXXI, n ${ }^{\circ} 179$, p. 142. Voir aussi lettres du 28 janvier et du 2 février 1902 à A. Linaker, O.C., XIX/1, nos 297 et 298, pp. 442 s. 
difficile à lire, car ne comportant pas un mot de trop ${ }^{56}$. D'autres lettres attestent plus tard que Pareto avait discuté avec Roguin de certains points de son Traité de sociologie générale $e^{57}$.

Par la suite cependant, des désaccords se manifestèrent entre les deux hommes notamment à propos de la sociologie. Roguin, qui s'intéressait depuis longtemps à cette discipline ${ }^{58}$, ne partageait pas toutes les vues de Pareto, qui semble avoir réagi en lui déniant toute compétence en la matière. Un premier différend éclata en 1912, lors de la soutenance de thèse d'une des doctorantes de Pareto, la Polonaise Maria Kolabinska, sur la Circulation des élites en France. Il n'y a pas lieu d'exposer ici les détails de cette affaire bien connue ${ }^{59}$. Rappelons seulement que cette étudiante avait suivi son maître sur certains points qu'il savait contestés notamment par Millioud. Or Pareto, invoquant son état de santé, avait renoncé à se rendre à Lausanne pour la soutenance, confiée dès lors à un jury ne le comprenant pas et composé de Millioud, Roguin et Boninsegni, sous la présidence du professeur de littérature française Paul Sirven. Se doutant que Millioud en particulier risquait d'attaquer fortement certains aspects de la thèse, Pareto avait cependant demandé à deux de ses disciples, Pierre Boven et Stanislao Scalfati, d'y assister dans le public et de soutenir au besoin la candidate.

Bien que le doctorat ait finalement été accordé, la soutenance s'était mal passée: Millioud avait effectivement critiqué vivement le travail de la candidate. Boven avait alors demandé la parole pour la défendre et lire une lettre de Pareto. Sur quoi Millioud s'était considéré comme personnellement attaqué, avait soulevé des problèmes de procédure et menacé de quitter la salle. Bref, une soutenance comme on espère bien ne jamais en vivre ... Pareto semble avoir considéré que Roguin, qui avait aussi formulé des critiques, avait une part de responsabilité dans cette affaire. Il lui reprocha notamment d' "avoir attendu dix ans ... pour l'attaquer, choisissant prudemment pour cela une occasion où l'auteur du principe était absent $»^{60}$. C'est à la suite de cette affaire qu'il l'appelle dans certaines lettres à Boven «Mr R.» ou même «Pauvre M. Roguin ${ }^{61}$.

Au début de l'année suivante, les relations entre les deux hommes ${ }^{62}$ étaient cependant à nouveau bonnes et Pareto décidait d'ajouter sur les conseils de

${ }^{56}$ Lettre du 22 juillet 1895, O.C., XXVIII/2, no 194, pp. 426 ss.

57 Cf. notes 63 et 70 ci-après.

${ }^{58}$ Le Traité de droit comparé relève déjà la nécessité d'approches sociologiques, cf. Busino, Ernest Roguin e Vilfredo Pareto, art. cit., in O.C., XIX/2, p. 1239.

${ }^{59} \mathrm{Cf}$. Busino, Pareto à travers sa correspondance, art. cit., pp. 58 ss. Voir aussi O.C., XIX, Introduzione, Materiali per l'edizione dell'epistolario par Giovanni Busino, pp. 27 ss, la correspondance publiée dans O.C., XIX/2, n ${ }^{\text {os }} 731$ ss, pp. 765 ss et les lettres à Millioud et à Roguin concernant cette affaire publiées par Della Ferrera, Appendice, art. cit., pp. 343 ss.

${ }^{60} \mathrm{Cf}$. annexe à la lettre de Pareto à Boven du 25 mai 1912, O.C., XIX/2, nº 737, pp. $769 \mathrm{~s}$.

${ }^{61}$ Cf. notes 46 et 47 ci-dessus.

${ }^{62}$ En revanche, les relations entre Pareto et Millioud restèrent détestables dès lors. Millioud, dont il était prévu qu'il traduirait en français le cours de sociologie de Pareto, lui renvoya son manuscrit et c'est finalement Boven qui se chargea de cette traduction, cf. Busino, Vilfredo Pareto à travers sa correspondance, art. cit., pp. 62 ss. Millioud s'efforça vainement de s'opposer à la célébration d'un jubilé Pareto, en soulignant combien, depuis des années, ce dernier se consacrait en réalité peu à l'enseignement, cf. ibidem, p. 65 s.; Biaudet, Préface, art. cit., in O.C., XX, p. 16. C'est en vain que 
Roguin des traductions d'auteurs latins dans la future version française de son Traité de sociologie générale ${ }^{63}$. Au printemps 1914, en lui confirmant une invitation à venir à Céligny, il lui donnait des nouvelles domestiques et lui écrivait «vous verrez que nous n'auront pas la guerre maintenant; les puissances financières veulent les dépenses pour la guerre, mais non la guerre ${ }^{64}$, ce qui prouve qu'on peut être un grand savant et un mauvais prophète ... En 1915, Pareto évoquait avec philosophie leurs divergences, en se déclarant heureux de voir que pour certains points, Roguin et lui étaient du même avis «bien que (...), je le crains fort, nous ne nous entendions en rien sur la Sociologie ${ }^{65}$, ce qui ne les empêchera pas de s'en entretenir parfois ${ }^{66}$.

Une autre période de tension intervint avec les préparatifs du jubilé des 25 ans d'enseignement de Pareto à Lausanne, pour lequel des cérémonies étaient prévues en juillet 1917. Lorsque Pareto appris que la Faculté de droit voulait confier à Roguin le soin de présenter sa sociologie, il s'inquiéta: pouvait-il admettre que la présentation de cette partie de son œuvre soit confiée à quelqu'un qui ne partageait pas toutes ses vues en la matière? Il écrivit même au doyen de l'époque, André Mercier $^{67}$, en parlant de «graves dissensions qui existaient entre Monsieur Roguin et [lui], précisément au sujet de [s]a Sociologie, qu'il appréciait sévèrement»; pour ce motif, indiquait-il: «Depuis longtemps ... j'évite soigneusement de parler de sociologie avec Monsieur Roguin, dont je désire conserver l'amitié »; il rappelait les incidents de la soutenance Kolabinska et suggérait qu'il valait peut-être mieux annuler les cérémonies prévues, en prétextant d'un renvoi dû aux circonstances internationales. Finalement, il faudra que Roguin fasse à Céligny une longue visite au futur jubilaire pour l'apaiser. Le 24 juin 1917, Pareto écrit au doyen Mercier que «Monsieur Roguin est venu ici hier ... et d'après ce qu'il a dit, toute crainte d'un conflit est écartée ${ }^{68}$. Il se déclara après coup satisfait de la manière dont Roguin avait présenté son travail ${ }^{69}$. Au début de 1919, il discutait avec lui de multiples questions en relation avec son Traité de Sociologie générale $^{70}$, dont l'édition française était entre-temps parue.

Une dernière facette des relations entre Roguin et Pareto mérite d'être brièvement évoquée, soit le rôle que le premier a joué pour mettre en relations le second avec diverses personnes en Suisse romande. On a vu que dès 1893 il l'avait fait entrer au Cercle littéraire. Peut-être est-ce à lui que Pareto doit d'avoir été chargé

Roguin tenta alors de réconcilier les deux hommes, tentatives dont Pareto finit d'ailleurs par s'irriter un peu, tout en affirmant que le refus d'une telle réconciliation ne provenait pas de lui, cf. lettre de Pareto à Pantaleoni du 4 mai 1917, O.C., XXVIII/3, nº 649, pp. 207 ss.

${ }^{63}$ Cf. lettre de Pareto à Boven du 17 février 1913, O.C., XIX/2, nº 801, p. 816.

${ }^{64} \mathrm{Cf}$. lettre de Pareto à Roguin du 20 mars 1914, O.C., XIX/2, no 879, pp. $864 \mathrm{~s}$.

${ }^{65}$ Cf. lettre de Pareto à Roguin d'août 1915, O.C., XXXI, nº 378, p. 265.

${ }^{66} \mathrm{Cf}$. par exemple note 70 ci-après.

${ }^{67}$ Cf. lettre du 19 juin 1917, O.C., XXX, no 390, p. 580 ss.

${ }^{68}$ Cf. O.C., XXX, no 392 , p. 587.

${ }^{69}$ Cf. Biaudet, Préface, art. cit., in O.C., XX, p. 17; Busino, Roguin e Pareto, art. cit., in O.C., XIX/2, p. 1245.

${ }^{70}$ Cf. lettre de Pareto à Roguin du 30 janvier 1919, O.C., XIX/2, nº 1109, pp. 1009 s. 
dès 1895 , alors qu'il était encore un nouveau venu à Lausanne, d'aider l'assurance-vie La Fraternité à se réorganiser ${ }^{71}$.

C'est par Roguin également que Pareto entra en contact à la fin de 1896 avec le professeur de philosophie genevois Adrien Naville, dont ledit Roguin était le beau-frère ${ }^{72}$. Tous deux eurent dès lors de fructueuses relations et échangèrent une correspondance régulière entre 1897 et $1923^{73}$.

Au total, Roguin a ainsi tenu une place non négligeable dans la vie de Pareto depuis sa venue à Lausanne et c'est d'ailleurs sans doute dans les travaux relatifs à Pareto qu'il reste le plus évoqué aujourd'hui. Le personnage et l'œuvre méritent cependant qu'on s'y intéresse pour eux-mêmes et pourraient même justifier une monographie en français, tenant notamment compte des importants fonds d'archives qui existent à leur sujet et attendent d'être exploités ${ }^{74}$.

${ }^{71}$ Cf. Giovanni Busino, Ricerche di Vilfredo Pareto in materia d'assicurazioni, in O.C., XIX/2, pp. 1173 ss; ibidem, Roguin e Pareto, art. cit., O.C., XIX/2, p. 1238.

${ }^{72}$ Cf. Busino, Roguin e Pareto, art. cit., in O.C., XIX/2 p. 1239. Adrien Naville avait en effet épousé Isabelle Roguin (1853-1933), sœur cadette d'Ernest.

${ }^{73}$ Sur les relations entre Pareto et Naville, cf. notamment Giovanni Busino, Pareto au Congrès international de philosophie de Genève, 1904, in «Archiv für Geschichte der Philosophie », 45, 1963, pp. $33 \mathrm{~s}$. C'est notamment grâce à Naville que put être organisée la venue à l'université de Genève de Maffeo Pantaleoni, l'ami de toujours de Pareto, cf. ibidem, Maffeo Pantaleoni à l'Université de Genève, in O.C., XIX/2, pp. 1207 ss; ibidem, Roguin e Pareto, art. cit., O.C., XIX/2 p. 1239 et O.C., XXVIII/1, n ${ }^{\text {os }} 246$, pp. 498 ss. Dans le cadre des discussions déjà évoquées qui conduisirent en 1900 à une première réduction de ses tâches d'enseignement, cf. note 38 ci-dessus, le doyen Erman estimait même que Naville et Pantaleoni manœuvraient pour débaucher Pareto en lui offrant une chaire à Genève, cf. ibidem, Vilfredo Pareto à travers sa correspondance, art. cit., p. 48.

${ }^{74} \mathrm{Cf}$. Bibliothèque cantonale et universitaire, fonds Ernest Roguin (IS 4939), 44 cartons et Archives cantonales vaudoises, fonds de la famille Roguin (PP 51 D 52/1-7). 